\title{
Observation of the cosmic ray shadow of the Sun with the ANTARES neutrino telescope
}

A. Albert, ${ }^{1,2}$ M. André, ${ }^{3}$ M. Anghinolfi, ${ }^{4}$ G. Anton, ${ }^{5}$ M. Ardid, ${ }^{6}$ J.-J. Aubert, ${ }^{7}$ J. Aublin, ${ }^{8}$ B. Baret, ${ }^{8}$ S. Basa, ${ }^{9}$ B. Belhorma,${ }^{10}$ V. Bertin, ${ }^{7}$ S. Biagi, ${ }^{11}$ M. Bissinger, ${ }^{5}$ J. Boumaaza, ${ }^{12}$ M. Bouta, ${ }^{13}$ M. C. Bouwhuis,${ }^{14}$ H. Brânzaş, ${ }^{15}$ R. Bruijn,${ }^{14,16}$ J. Brunner, ${ }^{7}$ J. Busto, ${ }^{7}$ A. Capone, ${ }^{17,18}$ L. Caramete, ${ }^{15}$ J. Carr, ${ }^{7}$ S. Celli, ${ }^{17,18}$ M. Chabab,${ }^{19}$ T. N. Chau, ${ }^{8}$ R. Cherkaoui El Moursli, ${ }^{12}$ T. Chiarusi, ${ }^{20}$ M. Circella, ${ }^{21}$ A. Coleiro, ${ }^{8}$ M. Colomer-Molla ${ }^{8,22}$ R. Coniglione, ${ }^{11}$ P. Coyle, ${ }^{7}$ A. Creusot, ${ }^{8}$ A. F. Díaz, ${ }^{23}$ G. de Wasseige, ${ }^{8}$ A. Deschamps,${ }^{24}$ C. Distefano, ${ }^{11}$ I. Di Palma, ${ }^{17,18}$ A. Domi,${ }^{4,25}$ C. Donzaud, ${ }^{8,26}$ D. Dornic, ${ }^{7}$ D. Drouhin, ${ }^{1,2}$ T. Eberl, ${ }^{5}$ N. El Khayati, ${ }^{12}$ A. Enzenhöfer, ${ }^{7}$ A. Ettahiri, ${ }^{12}$ P. Fermani, ${ }^{17,18}$ G. Ferrara, ${ }^{11}$ F. Filippini, ${ }^{20,27}$ L. Fusco, ${ }^{8,7}$ P. Gay,${ }^{8,28}$ H. Glotin, ${ }^{29}$ R. Gozzini, ${ }^{22,5}$ K. Graf, ${ }^{5}$ C. Guidi, ${ }^{4,25}$ S. Hallmann, ${ }^{5}$ H. van Haren,${ }^{30}$ A. J. Heijboer, ${ }^{14}$ Y. Hello, ${ }^{24}$ J. J. Hernández-Rey, ${ }^{22}$ J. Höß1, ${ }^{5}$ J. Hofestädt ${ }^{5}{ }^{5}$ F. Huang, ${ }^{1}$ G. Illuminati, ${ }^{8,22}$ C. W. James ${ }^{31}$ M. de Jong, ${ }^{14,32}$ P. de Jong, ${ }^{14}$ M. Jongen, ${ }^{14}$ M. Kadler, ${ }^{33}$ O. Kalekin, ${ }^{5}$ U. Katz, ${ }^{5}$ N. R. Khan-Chowdhury, ${ }^{22}$ A. Kouchner, ${ }^{8,34}$ I. Kreykenbohm, ${ }^{35}$ V. Kulikovskiy, ${ }^{4,36}$ R. Lahmann, ${ }^{5}$ R. Le Breton,${ }^{8}$ D. Lefèvre, ${ }^{37}$ E. Leonora, ${ }^{38}$ G. Levi, ${ }^{20,27}$ M. Lincetto, ${ }^{7}$ D. Lopez-Coto, ${ }^{39}$ S. Loucatos, ${ }^{40,8}$ G. Maggi, ${ }^{7}$ J. Manczak, ${ }^{22}$ M. Marcelin, ${ }^{9}$ A. Margiotta, ${ }^{20,27}$ A. Marinelli, ${ }^{41}$

J. A. Martínez-Mora, ${ }^{6}$ R. Mele, ${ }^{41,42}$ K. Melis, ${ }^{14,16}$ P. Migliozzi, ${ }^{41}$ M. Moser, ${ }^{5}$ A. Moussa, ${ }^{43}$ R. Muller, ${ }^{14}$ L. Nauta, ${ }^{14}$ S. Navas, ${ }^{39}$ E. Nezrii, ${ }^{9}$ C. Nielsen, ${ }^{8}$ A. Nuñez-Castiñeyra, ${ }^{7,9}$ B. O’ Fearraigh, ${ }^{14}$ M. Organokov, ${ }^{1}$ G. E. Păvălaş, ${ }^{15}$

C. Pellegrino,${ }^{20,44,45}$ M. Perrin-Terrin, ${ }^{7}$ P. Piattelli, ${ }^{11}$ C. Poirè, ${ }^{6}$ V. Popa,${ }^{15}$ T. Pradier, ${ }^{1}$ N. Randazzo, ${ }^{38}$ S. Reck, ${ }^{5}$

G. Riccobene, ${ }^{11}$ A. Romanov $\odot,{ }^{36,46, *}$ A. Sánchez-Losa, ${ }^{21}$ D. F. E. Samtleben, ${ }^{14,32}$ M. Sanguineti $\odot,{ }^{4,25, \uparrow}$ P. Sapienza, ${ }^{11}$ J. Schnabel, ${ }^{5}$ F. Schüssler, ${ }^{40}$ M. Spurio, ${ }^{20,27}$ Th. Stolarczyk ${ }^{40}$ B. Strandberg, ${ }^{14}$ M. Taiuti, ${ }^{4,25}$ Y. Tayalati, ${ }^{12}$ T. Thakore, ${ }^{22}$ S. J. Tingay, ${ }^{31}$ B. Vallage, ${ }^{40,8}$ V. Van Elewyck, ${ }^{8,34}$ F. Versari, ${ }^{20,27,8}$ S. Viola, ${ }^{11}$ D. Vivolo, ${ }^{41,42}$ J. Wilms, ${ }^{35}$ A. Zegarelli, ${ }^{17,18}$ J. D. Zornoza, ${ }^{22}$ and J. Zúñiga ${ }^{22}$

(ANTARES Collaboration)

\author{
${ }^{1}$ Université de Strasbourg, CNRS, IPHC UMR 7178, F-67000 Strasbourg, France \\ ${ }^{2}$ Université de Haute Alsace, F-68200 Mulhouse, France \\ ${ }^{3}$ Technical University of Catalonia, Laboratory of Applied Bioacoustics, Rambla Exposició, \\ 08800 Vilanova i la Geltrú, Barcelona, Spain \\ ${ }^{4}$ INFN_Sezione di Genova, Via Dodecaneso 33, 16146 Genova, Italy \\ ${ }^{5}$ Friedrich-Alexander-Universität Erlangen-Nürnberg, Erlangen Centre for Astroparticle Physics, \\ Erwin-Rommel-Str. 1, 91058 Erlangen, Germany \\ ${ }^{6}$ Institut d'Investigació per a la Gestió Integrada de les Zones Costaneres (IGIC)—Universitat Politècnica \\ de València. C/ Paranimf 1, 46730 Gandia, Spain \\ ${ }^{7}$ Aix Marseille Univ, CNRS/IN2P3, CPPM, Marseille, France \\ ${ }^{8}$ Université de Paris, CNRS, Astroparticule et Cosmologie, F-75006 Paris, France \\ ${ }^{9}$ Aix Marseille Univ, CNRS, CNES, LAM, Marseille, France \\ ${ }^{10}$ National Center for Energy Sciences and Nuclear Techniques, B.P.1382, R. P.10001 Rabat, Morocco \\ ${ }^{11}$ INFN_Laboratori Nazionali del Sud (LNS), Via S. Sofia 62, 95123 Catania, Italy \\ ${ }^{12}$ University Mohammed $V$ in Rabat, Faculty of Sciences, \\ 4 av. Ibn Battouta, B.P. 1014, R.P. 10000 Rabat, Morocco \\ ${ }^{13}$ University Mohammed I, Laboratory of Physics of Matter and Radiations, \\ B.P. 717, Oujda 6000, Morocco \\ ${ }^{14}$ Nikhef, Science Park, Amsterdam, The Netherlands \\ ${ }^{15}$ Institute of Space Science, RO-077125 Bucharest, Măgurele, Romania \\ ${ }^{16}$ Universiteit van Amsterdam, Instituut voor Hoge-Energie Fysica, \\ Science Park 105, 1098 XG Amsterdam, The Netherlands \\ ${ }^{17}$ INFN-Sezione di Roma, P.le Aldo Moro 2, 00185 Roma, Italy \\ ${ }^{18}$ Dipartimento di Fisica dell'Università La Sapienza, P.le Aldo Moro 2, 00185 Roma, Italy \\ ${ }^{19}$ LPHEA, Faculty of Science-Semlali, Cadi Ayyad University, P.O.B. 2390, Marrakech, Morocco \\ ${ }^{20}$ INFN-Sezione di Bologna, Viale Berti-Pichat 6/2, 40127 Bologna, Italy \\ ${ }^{21}$ INFN-Sezione di Bari, Via E. Orabona 4, 70126 Bari, Italy \\ ${ }^{22}$ IFIC-Instituto de Física Corpuscular (CSIC-Universitat de València) c/ Catedrático José Beltrán, \\ 2 E-46980 Paterna, Valencia, Spain \\ ${ }^{23}$ Department of Computer Architecture and Technology/CITIC, University of Granada, \\ 18071 Granada, Spain \\ ${ }^{24}$ Géoazur, UCA, CNRS, IRD, Observatoire de la Côte d'Azur, Sophia Antipolis, France
}




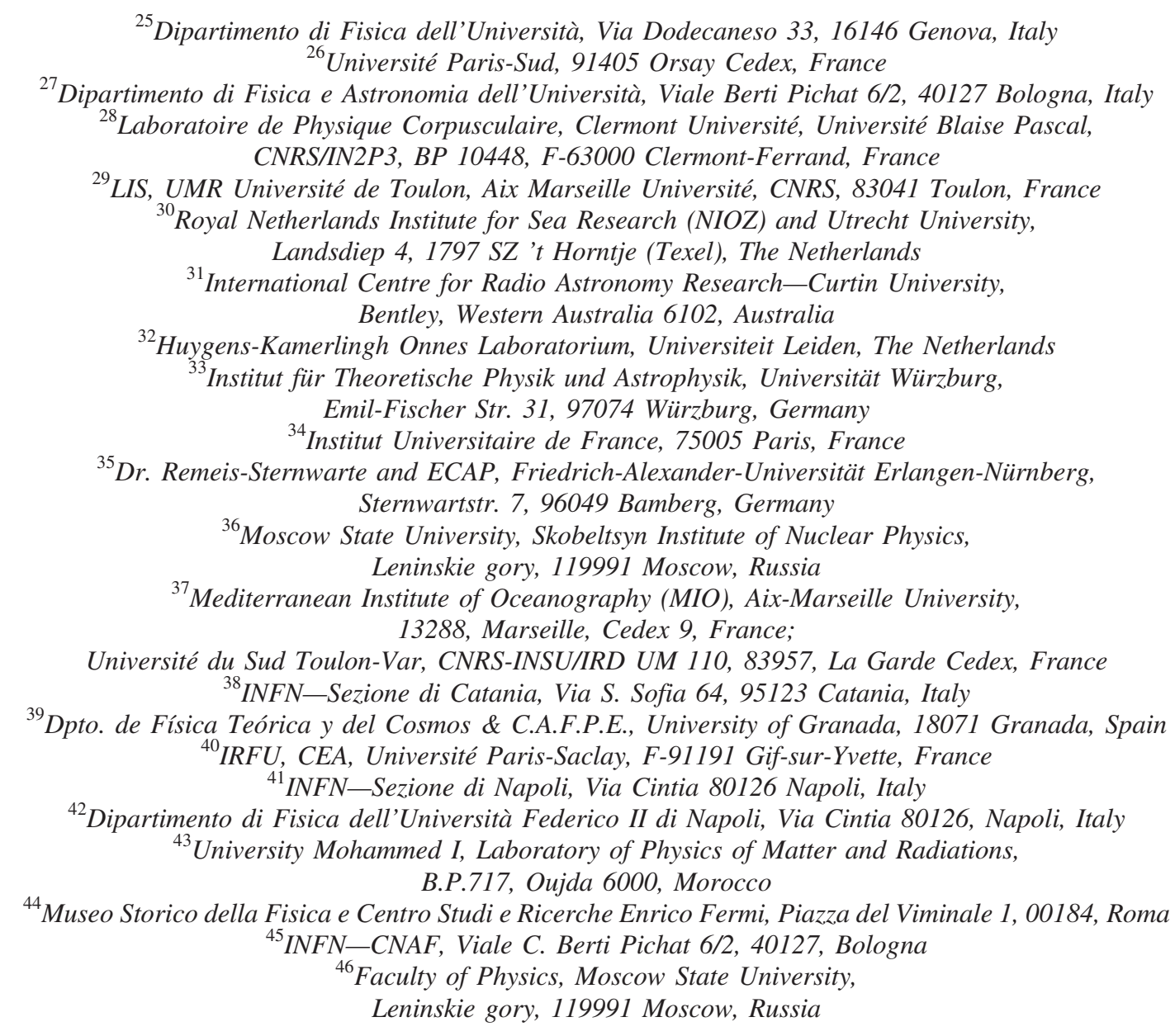

(Received 3 July 2020; revised 27 October 2020; accepted 30 November 2020; published 28 December 2020)

The ANTARES detector is an undersea neutrino telescope in the Mediterranean Sea. The search for pointlike neutrino sources is one of the main goals of the ANTARES telescope, requiring a reliable method to evaluate the detector angular resolution and pointing accuracy. This work describes the study of the Sun "shadow" effect with the ANTARES detector. The shadow is the deficit in the atmospheric muon flux in the direction of the Sun caused by the absorption of the primary cosmic rays. This analysis is based on the data collected between 2008 and 2017 by the ANTARES telescope. The observed statistical significance of the Sun shadow detection is $3.7 \sigma$, with an estimated angular resolution of $0.59^{\circ} \pm 0.10^{\circ}$ for downward-going muons. The pointing accuracy is found to be consistent with the expectations and no evidence of systematic pointing shifts is observed.

DOI: $10.1103 /$ PhysRevD.102.122007

\section{INTRODUCTION}

Charged cosmic rays (CRs) (mainly protons), $\gamma$-rays, and neutrinos represent relevant probes for high-energy astrophysical research. However, $\gamma$-rays with energies higher than few $\mathrm{TeV}$ interact with the infrared and the cosmic

\footnotetext{
*Corresponding author. andrey.romanov@ge.infn.it

${ }^{\dagger}$ Corresponding author. matteo.sanguineti@unige.it
}

microwave background producing electron-positron pairs. Charged CRs are deflected by cosmic magnetic fields, and it is almost impossible to identify their origin through the measurement of their arrival direction. Moreover, the structure of galactic magnetic fields is so complex that the distribution of galactic CRs is almost isotropic near the Earth. Neutrinos have properties which allow to observe and study the Universe in a unique way. They can propagate from their sources to the Earth without changing trajectory and with small probability of being absorbed. 
The ANTARES undersea neutrino telescope [1] is primarily designed for the detection of neutrino pointlike sources and both the pointing accuracy and the angular resolution of the detector are important for the evaluation of the telescope performance.

The interaction of primary CRs in the atmosphere produces secondary downward-going muons that can be detected in the undersea detector. However, the CRs could be absorbed by the Moon and the Sun leading to a deficit in the atmospheric muon flux in the directions of these celestial bodies. This effect has been observed by several experiments: CYGNUS [2], TIBET [3], BUST [4], CASA [5], MACRO [6], SOUDAN [7], ARGO-YBG [8], HAWC [9], MINOS [10], and also IceCube [11]. A Moon shadow analysis with the ANTARES telescope, corresponding to a total live time of 3128 days, has also been published [12].

This work presents the Sun shadow analysis using the ANTARES 2008-2017 data sample, corresponding to a total detector live time of 2925 days. The analysis is based on $2.6 \times 10^{6}$ events reconstructed as downward-going muons with the standard ANTARES reconstruction chain [13].

The paper is organized as follows: in Sec. II, the ANTARES neutrino telescope is described; the Sun shadow analysis and the obtained results are presented in Sec. III; finally, the conclusions are summarized in Sec. IV.

\section{THE ANTARES NEUTRINO TELESCOPE}

The ANTARES undersea neutrino telescope is taking data in its final configuration since 2008. It is located in the Mediterranean Sea, $40 \mathrm{~km}$ offshore from Toulon (France) at $42^{\circ} 48 \mathrm{~N}$ latitude and $6^{\circ} 10 \mathrm{E}$ longitude. The detector consists of 12 lines, each is about $450 \mathrm{~m}$ long. Each line comprises 25 storeys with three 10 -inch photomultiplier tubes (PMTs) inside pressure resistant glass spheres (the optical modules). The first instrumented storey is located $100 \mathrm{~m}$ above the seabed. The distance between storeys is $14.5 \mathrm{~m}$ and the distance between two lines is about $65 \mathrm{~m}$. The lines are connected to a junction box that links the detector to the shore station through an electro-optical cable about $40 \mathrm{~km}$ long.

A relativistic muon induces Cherenkov photons when traveling through the water, which are detected by the PMTs producing a signal (hit) [1]. The PMTs face $45^{\circ}$ downward in order to optimize the detection of light from upward-going particles. The set of hits detected within a certain time window is called event. If the hits of one event satisfy spacetime causality, the event is identified as a muon candidate $[13,14]$. The reconstruction of the tracks is based on the probability density function of the arrival times of photons at the PMTs.

\section{THE SUN SHADOW ANALYSIS}

The ANTARES telescope can detect only downwardgoing atmospheric muons because the upward-going ones are absorbed by the Earth. The energy threshold of muons at the sea surface level that can reach the detector is about $500 \mathrm{GeV}$ [15]. In this energy range, the direction of primary CRs may be assumed as collinear with the secondary muons. Even though the solar magnetic field is not expected to introduce a systematic shift in the pointing accuracy derived using the Sun shadow effect, it is expected that its influence can lead to a blurring of the shadow [11]. Therefore, the primaries that are blocked by the Sun lead to a deficit in the atmospheric muon flux in the direction of the Sun.

The analysis is performed in three steps. The first one is the data selection optimization which provides the best sensitivity for the observation of the deficit of events from the direction of the Sun. The second step provides the estimation of the angular resolution of the detector for the reconstructed downward-going events. And in the third step a possible shift of the Sun shadow center with respect to the nominal Sun position is investigated using a twodimensional approach.

\section{A. The data selection optimization}

A Monte Carlo (MC) simulation is produced and exploited in order to optimize the event selection criteria of the analysis. The simulation features downward-going muon events which are generated at the detector level with the MUPAGE code [16]. MUPAGE is based on parametric formulas that allow to calculate the flux and the angular distribution of underwater muon bundles, taking into account the muon multiplicity and the energy spectrum. Muons are generated on the surface of a cylinder (can) surrounding the active volume of the detector, $650 \mathrm{~m}$ high, with a radius of $290 \mathrm{~m}$. The simulation includes the propagation of the muons in the instrumented volume, the induced emission of Cherenkov light, the light propagation to the optical modules, and the digitized response of the PMTs [17]. In order to reproduce the time variability of the detector conditions, the MC sample is subdivided in batches corresponding to the actual data-taking periods (run-by-run MC simulation [18]). The trade-off between the accuracy of the simulations and CPU time, exploited to produce the MC sample, limited the MC muon statistics to $1 / 3$ of the actual expected one. In order to enlarge the statistics of MC simulation, the additional zones approach was performed: since the muon generation is produced on a full-sky base, regions of the sky with the same occupancy as of the Sun region can be exploited, where the MC sample can be increased. Additional zones are obtained artificially by shifting the Sun position by 2, 4, 6, 8, 12, 14, 16, 18, 20, and 22 hours. Therefore, the whole statistics of MC considering 11 additional zones together with the Sun zone is 4 times larger than the real data statistics.

Since the atmospheric muon flux is not uniform, the distribution of muons depends also on the Sun elevation angle at the moment of muon detection. Statistics of the 
events significantly decreases for muon tracks close to the horizon; for this reason, a cut on the Sun elevation angle is applied: $\theta_{\text {Sun }}>15^{\circ}$.

The quality of the reconstructed tracks is determined by two parameters: the likelihood-wise parameter, $\Lambda$, and the angular error estimator of the reconstructed direction, $\beta$ [19]. In order to determine for which set of cut values on $\Lambda$ and $\beta$ the sensitivity of the Sun shadow detection is maximal, the hypothesis test approach is used. The null hypothesis $H_{0}$ corresponds to the absence of the Sun shadowing effect, while the $H_{1}$ hypothesis is compliant with the presence of this phenomenon. Then two different MC samples are generated. According to the null hypothesis, in the first sample, the Sun shadow effect is not introduced in the simulation; according to the $H_{1}$ hypothesis, in the second sample the Sun shadow effect is obtained by removing all the muons generated within the Sun disk, assuming a radius of $0.26^{\circ}$. For each sample, the distribution of events as a function of the angular distance from the Sun, up to $10^{\circ}$, is produced. Such a histogram is subdivided into 25 bins with size $\Delta \delta=0.4^{\circ}$. Each bin corresponds to a concentric ring with increasing radius centered on the Sun position. The content of each bin is normalized to the corresponding area of the ring, resulting in an event density.

Assuming that the event population in each bin asymptotically follows a Gaussian probability distribution, the test statistic is calculated under the above mentioned two hypotheses as a $\chi^{2}$ difference, resulting in $\lambda_{0}$ and $\lambda_{1}$,

$$
\begin{aligned}
& \lambda_{0}=\sum_{i=1}^{N_{\text {bins }}}\left[\frac{\left(n_{0}^{i}-\mu_{i}\right)^{2}}{\sigma_{\mu, i}^{2}}-\frac{\left(n_{0}^{i}-\nu_{i}\right)^{2}}{\sigma_{\nu, i}^{2}}\right], \\
& \lambda_{1}=\sum_{i=1}^{N_{\text {bins }}}\left[\frac{\left(n_{1}^{i}-\mu_{i}\right)^{2}}{\sigma_{\mu, i}^{2}}-\frac{\left(n_{1}^{i}-\nu_{i}\right)^{2}}{\sigma_{\nu, i}^{2}}\right],
\end{aligned}
$$

with $\mu_{i}\left(\nu_{i}\right)$ the expected number of events in the ith bin under $H_{1}\left(H_{0}\right)$ hypothesis, $\sigma_{\mu, i}\left(\sigma_{\nu, i}\right)$ the error in the ith bin under $H_{1}\left(H_{0}\right)$. The values of $n_{1}\left(n_{0}\right)$ are derived according to a Poisson distribution with expectation values equal to $\mu_{i}\left(\nu_{i}\right)$. A total of $10^{6}$ pseudoexperiments are generated to build the distribution of the test statistic.

The hypothesis test procedure is repeated for different sets of cut values on $\Lambda$ and $\beta$ to maximize the sensitivity to the Sun shadow detection (Fig. 1). Figure 2 shows the estimation of the sensitivity. It is evaluated through the computation of the p-value of the $\lambda_{0}$ distribution (null hypothesis, $H_{0}$ ) corresponding to the median of the $\lambda_{1}$ distribution, for which $50 \%$ of the pseudo-experiments under the $H_{1}$ hypothesis (presence of the Sun shadow) are correctly identified. For the optimized values of $\Lambda$ and $\beta$, the $\mathrm{p}$-value is equal to $7.4 \times 10^{-4}$, corresponding to a significance of $3.4 \sigma$.

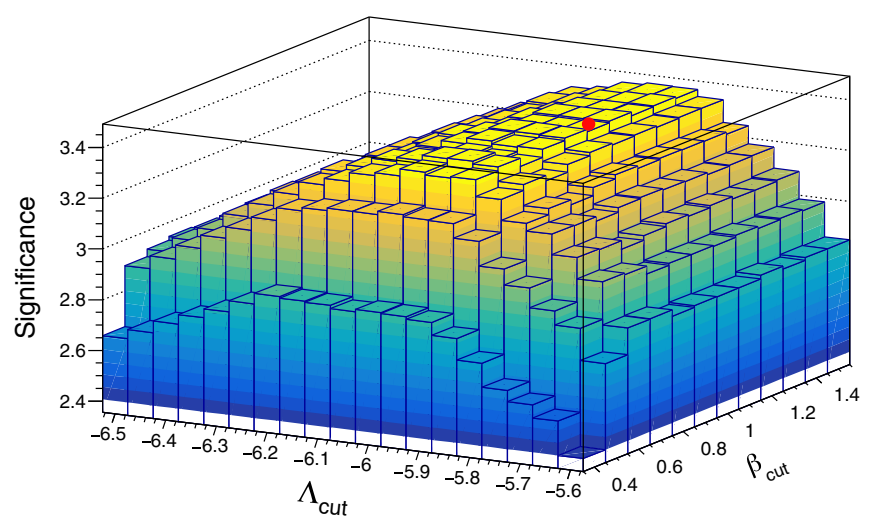

FIG. 1. Expected statistical significance of the Sun shadow detection during the period from 2008 to 2017 based on MC simulations, as a function of cut values on $\Lambda$ and $\beta\left(\Lambda_{\text {cut }}\right.$ and $\left.\beta_{\text {cut }}\right)$. The red point represents the selected set of cut values $\left(\Lambda_{\text {cut }}=-5.9\right.$ and $\left.\beta_{\text {cut }}=1.1^{\circ}\right)$. The expected significance for the selected set of cut values is $3.4 \sigma$.

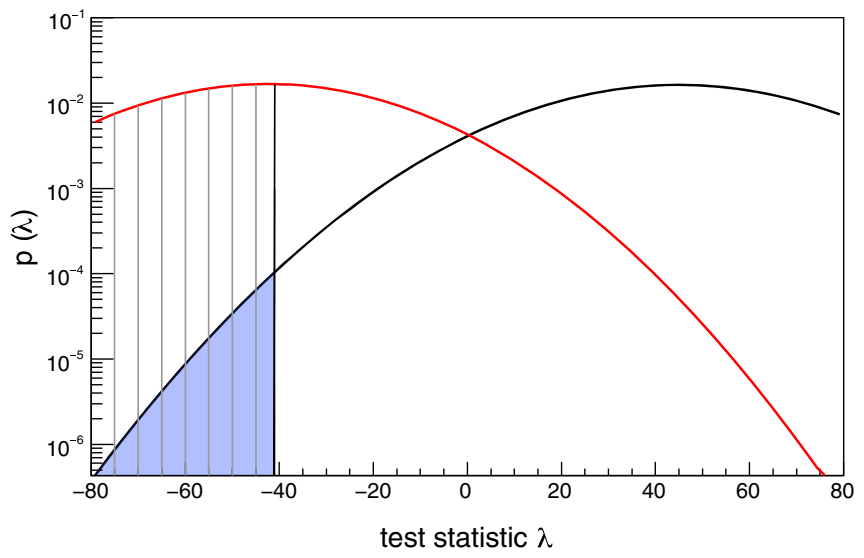

FIG. 2. Distribution of the test statistic $\lambda$ for the two hypotheses, $H_{0}$ (black curve) and $H_{1}$ (red curve), obtained for the optimized set of cut values $\left(\Lambda_{\text {cut }}=-5.9\right.$ and $\left.\beta_{\text {cut }}=1.1^{\circ}\right)$. The dashed area represents the fraction of pseudo-experiments $(50 \%)$ where $H_{1}$ hypothesis is correctly identified. The colored area corresponds to the expected median significance $(3.4 \sigma)$ to reject the $H_{0}$ hypothesis in favor of the $H_{1}$ hypothesis.

It is found that the sensitivity is almost constant for $-6.0<\Lambda_{\text {cut }}<-5.9$ and $0.6^{\circ}<\beta_{\text {cut }}<1.5^{\circ}$. In this parameter space, a particular set of cut values is chosen: $\Lambda_{\text {cut }}=$ -5.9 and $\beta_{\text {cut }}=1.1^{\circ}$. For this set of cut values, the muon density far from the Sun position is flat; this condition is required in the data significance estimation approach that will be described below in Sec. III B.

\section{B. The angular resolution estimation and significance of the results}

The reconstructed events from the 2008 to 2017 ANTARES data sample are selected with the optimized cut values described above, providing $6.5 \times 10^{5}$ events. The data event density distribution is produced in the same 


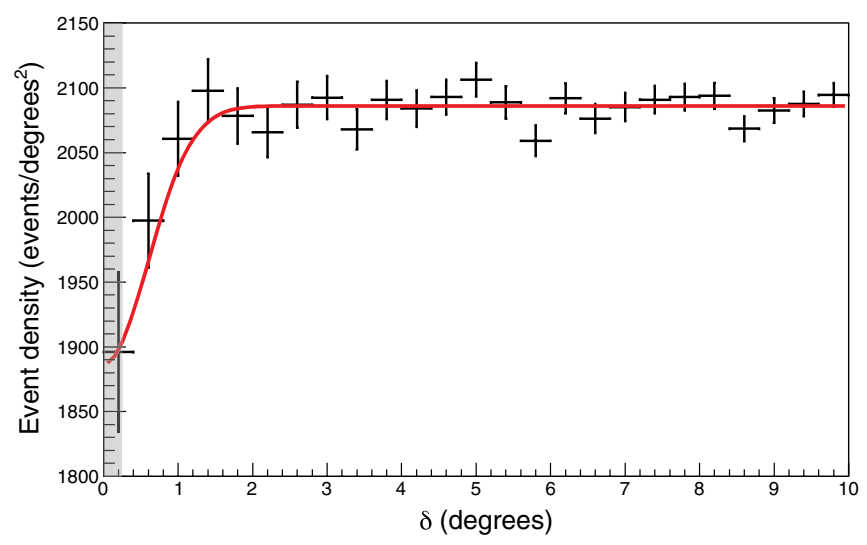

FIG. 3. The muon event density as a function of the angular distance $\delta$ from the Sun center based on the data sample taken in period 2008-2017 fitted with Eq. (2) (red line). The shaded area corresponds to the Sun angular radius $\left(0.26^{\circ}\right)$.

way as for the MC events described above in the hypothesis test procedure.

In order to estimate the angular resolution of the detector for downward-going muons, the data histogram is fitted with the following function [12] (red line in Fig. 3):

$$
f(\delta)=\frac{d N}{d \Omega}=k\left(1-\frac{R_{\mathrm{Sun}}^{2}}{2 \sigma_{\mathrm{res}}^{2}} e^{-\frac{\delta^{2}}{2 \sigma_{\mathrm{res}}}}\right)
$$

where $\Omega$ is the solid angle of the concentric ring around the Sun center, $k$ is the average muon event density in the $H_{0}$ hypothesis, the value of $k$ from the fit is $2086 \pm 2.6, R_{\text {Sun }}$ is the average angular radius of the Sun $\left(0.26^{\circ}\right)$, and $\sigma_{\text {res }}$ is the width of the Gaussian dip. The number of absorbed events in the Sun shadow dip is $N_{\text {abs }}=k \pi R_{\text {Sun }}^{2}=443 \pm 0.6$. The average muon event density obtained in the ANTARES Moon shadow study is $2376 \pm 3$ [12] and hence the number of absorbed events is $505 \pm 0.6$. The average muon event density in the Sun analysis is smaller with respect to the Moon analysis since the quality category to include a run has been slightly changed and a sample of runs with a tighter quality selection is chosen for the current analysis.

The value of $\sigma_{\text {res }}$ from the fit is $0.59^{\circ} \pm 0.10^{\circ}$. The goodness of the fit is found to be $\chi^{2} /$ d.o.f. $=19.6 / 23$.

Pseudo-experiments are used to evaluate the actual effect of a finite-size radius of the Sun. Several event densities are produced and convoluted with a step function representing the Sun radius assuming different detector angular resolutions. The discrepancies obtained between the assumed detector angular resolutions and the fitted values of the Gaussian width are below $10 \%$ for the assumed angular resolution values above $0.35^{\circ}$, i.e., negligible with respect to the statistical uncertainty. Therefore, the obtained value of $\sigma_{\text {res }}$ can be treated as the angular resolution of the telescope for downward-going muons.
The angular resolution value $\sigma_{\text {res }}$ is compatible with the one obtained in the ANTARES Moon shadow analysis $\left(0.73^{\circ} \pm 0.14^{\circ}\right)$, with a $3.5 \sigma$ significance of lunar detection [12].

The statistical significance of the result is estimated using the hypothesis test approach. For the $H_{0}$ hypothesis, no shadowing effect is assumed. Under this hypothesis, the data event density in Fig. 3 is fitted with the function which has one free parameter, $k$,

$$
\frac{d N}{d \Omega}=k
$$

The corresponding $\chi^{2}$ value is $\chi_{0}^{2}=33.5$. The $H_{1}$ hypothesis corresponds to the presence of the shadowing effect according to Eq. (2). The corresponding deviation of the data with respect to the $H_{0}$ hypothesis is computed by means of the test statistics: $-\lambda=\chi_{0}^{2}-\chi_{1}^{2}$, which follows a $\chi^{2}$ distribution with 1 degree of freedom. A significance of $3.7 \sigma$ is found. According to the MC pseudo-experiments, the probability to obtain such value of significance or higher is $37 \%$.

As reported by the IceCube Collaboration [11], the primary CRs can be influenced by the Sun magnetic field which can lead to the blurring of the shadow. In order to study the influence of this effect, the data sample is divided into two samples with roughly equal statistics. The first one covers the period from the middle of 2008 to the middle of 2011, when the Sun activity was in the lower half, while the second one covers the period from the middle of 2011 to the end of 2015, when the Sun activity was in the higher half. The statistical significance of the Sun shadow observation is almost the same in both data samples, $2.6 \sigma$ and $2.5 \sigma$ for the first and the second data samples, respectively, the spreading of the dip is also compatible within the statistical uncertainties. This is compatible with the results obtained in the other experiments since the statistics of the data sample is insufficient to obtain significative conclusions.

\section{Absolute pointing}

The procedure for the estimation of the pointing accuracy of the Sun shadow detection follows that used for the ANTARES Moon shadow study [12]. The distribution of events which satisfies the selection criteria described previously is projected in a two-dimensional histogram as a function of $x=\left(\alpha_{\mu}-\alpha_{\text {Sun }}\right) \times \cos \left(h_{\mu}\right)$ and $y=h_{\mu}-h_{\text {Sun }}$, where $\alpha_{\mu}, \alpha_{\text {Sun }}$ are the azimuthal coordinates and $h_{\mu}, h_{\text {Sun }}$ are the elevation angles of the reconstructed track and the Sun, respectively. The histogram range is $\left[-10^{\circ}, 10^{\circ}\right]$ for both $x$ and $y$, and it is divided in a grid of $0.4^{\circ} \times 0.4^{\circ}$ squared bins.

For the determination of a possible shift of the Sun shadow center with respect to the nominal Sun position, the following approach is used. Since the atmospheric muon flux depends mainly on the elevation angle, in the $H_{0}$ 


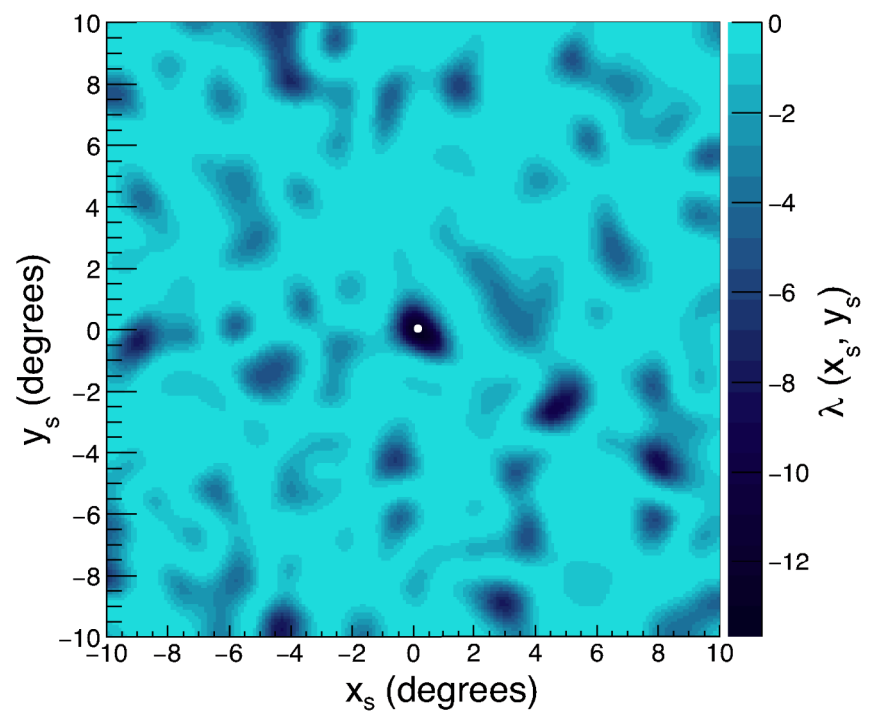

FIG. 4. The distribution of the test statistic values around the nominal Sun position $O \equiv\left(0^{\circ}, 0^{\circ}\right)$. The minimum value $\lambda_{\min }=$ -13.7 is found at $\left(0.2^{\circ}, 0^{\circ}\right)$ point (white dot).

hypothesis (no shadowing effect), the background distribution is approximated with a second degree polynomial,

$$
p_{2}(x, y ; \mathbf{k})=k_{0}+k_{1} x+k_{2} x^{2}+k_{3} y+k_{4} y^{2} .
$$

In the $H_{1}$ hypothesis (presence of the shadowing effect), the data distribution is approximated with a function obtained by subtracting from $p_{2}(x, y ; \mathbf{k})$ a two-dimensional Gaussian function,

$$
G\left(x, y ; A_{\mathrm{sh}}, x_{s}, y_{s}\right)=\frac{A_{\mathrm{sh}}}{2 \pi \sigma_{\mathrm{res}}^{2}} e^{-\frac{\left(x-x_{s}\right)^{2}+\left(y-y_{s}\right)^{2}}{2 \sigma_{\mathrm{res}}^{2}}},
$$

where $A_{\mathrm{sh}}$ is the amplitude of the deficit caused by the Sun shadow (free parameter), $\left(x_{s}, y_{s}\right)$ is the assumed position of the Sun. The width of the Gaussian function is assumed to be the same in both dimensions, so that $\sigma_{x}=\sigma_{y} \equiv \sigma_{\text {res }}$, and $\sigma_{\text {res }}$ is fixed to the value of the angular resolution defined in Eq. (2) and derived in the previous subsection.

In the pointing accuracy estimation, the Sun shadow center is assumed to be in the different points of the twodimensional histogram described above with a step size of $0.1^{\circ}$. The nominal Sun position is $O \equiv\left(0^{\circ}, 0^{\circ}\right)$. The test statistic function is then calculated for each assumed shift of the Sun position as

$$
\lambda\left(x_{s}, y_{s}\right)=\chi_{H_{1}}^{2}\left(x_{s}, y_{s}\right)-\chi_{H_{0}}^{2},
$$

where $\chi_{H_{0}}^{2}$ is the $\chi^{2}$ value obtained from the fit with Eq. (4), which is a constant value for all the bins of the histogram, and $\chi_{H_{1}}^{2}\left(x_{s}, y_{s}\right)$ is the $\chi^{2}$ value obtained from the fit with the function used to describe hypothesis $H_{1}, p_{2}(x, y ; \mathbf{k})-G\left(x, y ; A_{\mathrm{sh}}, x_{s}, y_{s}\right)$.

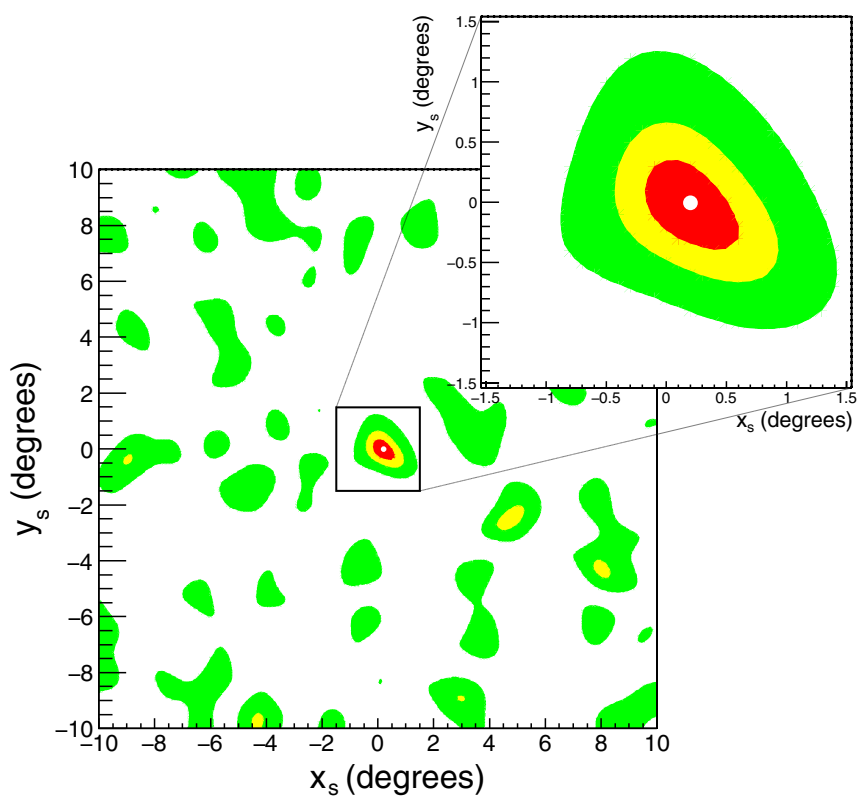

FIG. 5. Contours corresponding to different confidence levels (red: 68.27\%; yellow: 95.45\%; green: 99.73\%). The white dot indicates $\left(0.2^{\circ}, 0^{\circ}\right)$ point for which a minimum value of $\lambda_{\min }=-13.7$ is obtained.

Figure 4 shows the values of the test statistic as a function of the assumed Sun position, $\lambda\left(x_{s}, y_{s}\right)$. The minimum value of $\lambda\left(x_{s}, y_{s}\right)$ is found at $\left(0.2^{\circ}, 0^{\circ}\right)$ point and it is equal to $\lambda_{\min }=-13.7$. The corresponding fitted value of the Sun shadow dip amplitude is $A_{\min }=55 \pm 15$. The values of $\lambda\left(x_{s}, y_{s}\right)$ and $A_{\mathrm{sh}}$ for the nominal Sun position are $\lambda_{O}=-13.1$ and $A_{O}=54 \pm 15$. At each bin, $-\lambda$ follows the distribution of a $\chi^{2}$ with 1 degree of freedom, assuming $H_{0}$ as the true hypothesis. This allows the significance to reject the no-Sun hypothesis to be estimated. Considering $-\lambda_{O}$, a p-value of $3.1 \times 10^{-4}$ is obtained. The corresponding significance is $3.6 \sigma$.

The distribution of values of the test statistic $\lambda\left(x_{s}, y_{s}\right)$ can be interpreted as a bidimensional profile likelihood, with $A_{\mathrm{sh}}$ treated as the nuisance parameter. Therefore, the interval corresponding to a desired confidence level (CL) is obtained for $\lambda\left(x_{s}, y_{s}\right) \leq \lambda_{\text {cut }}=\lambda_{\min }+Q$, where $Q$ is the quantile for the joint estimation of two parameters, according to the values reported on Table 40.2 of [20]. Figure 5 shows the estimation of the confidence regions for $\mathrm{CL} \equiv\{68.27 \%, 95.45 \%, 99.73 \%\}$.

\section{CONCLUSIONS}

The evaluation of the angular resolution of the ANTARES detector is essential since one of the main goals of the telescope is the search for pointlike sources $[19,21,22]$.

This paper presents the observation of the Sun shadow with the ANTARES neutrino telescope. The analysis is 
based on the data taken in the period between 2008 and 2017 corresponding to a total detector live time of 2925 days.

The Sun shadow effect is studied by means of two complementary approaches which allow to determine the angular resolution for downward-going atmospheric muons and to verify the pointing performance of the detector. The shadow effect is observed with $3.7 \sigma$ statistical significance using the one-dimensional approach. The angular resolution for downward-going muons is found to be equal to $0.59^{\circ} \pm 0.10^{\circ}$. A better angular resolution is expected for upward-going events, as the PMTs of the detector are pointing $45^{\circ}$ below the horizon to maximize the light collection for upward-going neutrino-induced events.

The obtained angular resolution is compatible with the angular resolution found in the Moon shadow analysis with the ANTARES telescope $\left(0.73^{\circ} \pm 0.14^{\circ}\right)$ [12]

The influence of the Sun magnetic field on the primary CRs is investigated; however, the statistics is insufficient to obtain significative conclusions.

The resulting pointing accuracy of the Sun shadow detection is found to be consistent with the expectations.

\section{ACKNOWLEDGMENTS}

The authors acknowledge the financial support of the funding agencies: Centre National de la Recherche Scientifique, Commissariat à l'énergie atomique et aux énergies alternatives, Commission Européenne (FEDER fund and Marie Curie Program), Institut Universitaire de France, LabEx UnivEarthS (ANR-10-LABX-0023 and ANR-18-IDEX-0001), Région Île-de-France (DIM-ACAV), Région Alsace (contract CPER), Région ProvenceAlpes-Côte d'Azur, Département du Var and Ville de La Seyne-sur-Mer, France; Bundesministerium für Bildung und Forschung, Germany; Istituto Nazionale di Fisica Nucleare, Italy; Nederlandse organisatie voor Wetenschappelijk Onderzoek, the Netherlands; Council of the President of the Russian Federation for Young Scientists and Leading Scientific Schools supporting grants, Russia; Executive Unit for Financing Higher Education, Research, Development and Innovation (UEFISCDI), Romania; Ministerio de Ciencia, Innovación, Investigación y Universidades (MCIU): Programa Estatal de Generación de Conocimiento (refs. PGC2018-096663-B-C41, -A-C42, -B-C43, -B-C44) (MCIU/FEDER), Severo Ochoa Centre of Excellence and MultiDark Consolider (MCIU), Junta de Andalucía (refs. SOMM17/6104/UGR and A-FQM-053-UGR18), Generalitat Valenciana: Grisolía (ref. GRISOLIA/2018/119), Spain; Ministry of Higher Education, Scientific Research and Professional Training, Morocco. We also acknowledge the technical support of Ifremer, AIM and Foselev Marine for the sea operation and the CC-IN2P3 for the computing facilities.
[1] M. Ageron et al., Nucl. Instrum. Methods Phys. Res., Sect. A 656, 11 (2011).

[2] D. E. Alexandreas et al., Phys. Rev. D 43, 1735 (1991).

[3] M. Amenomori et al., Astropart. Phys. 28, 137 (2007).

[4] Yu. M. Andreyev, V. N. Zakidyshev, S. N. Karpov, and V. N. Khodov, Cosmic Res. (Transl. of Kosm. Issled.) 40, 559 (2002).

[5] A. Borione et al., Phys. Rev. D 49, 1171 (1994).

[6] M. Ambrosio et al., Astropart. Phys. 20, 145 (2003).

[7] J. H. Cobb et al., Phys. Rev. D 61, 092002 (2000).

[8] B. Bartoli et al., Phys. Rev. D 85, 022002 (2012).

[9] A. U. Abeysekara et al., Phys. Rev. D 97, 102005 (2018).

[10] P. Adamson et al., Astropart. Phys. 34, 457 (2011).

[11] M. G. Aartsen et al., Astrophys. J. 872, 133 (2019).

[12] A. Albert et al., Eur. Phys. J. C 78, 1006 (2018).
[13] S. Adrian-Martinez et al., J. Cosmol. Astropart. Phys. 03 (2013) 006.

[14] J. A. Aguilar et al., Astropart. Phys. 34, 652 (2011).

[15] Y. Becherini, A. Margiotta, M. Sioli, and M. Spurio, Astropart. Phys. 25, 1 (2006).

[16] G. Carminati, M. Bazzotti, A. Margiotta, and M. Spurio, Comput. Phys. Commun. 179, 915 (2008).

[17] H. Yepes-Ramírez et al., Nucl. Instrum. Methods Phys. Res., Sect. A 725, 203 (2013).

[18] L. A. Fusco and A. Margiotta, Eur. Phys. J. Web Conf. 116, 02002 (2016).

[19] A. Albert et al., Phys. Rev. D 96, 082001 (2017).

[20] P. A. Zyla et al. (Particle Data Group), Prog. Theor. Exp. Phys. 2020, 083C01 (2020).

[21] A. Albert et al., Astrophys. J. 892, 92 (2020).

[22] S. Adrian-Martinez et al., Astrophys. J. Lett. 786, L5 (2014). 